\title{
Stents coronaires
}

\section{0 ans de progrès médical}

Johanne Silvain ${ }^{1}$, Guillaume Cayla ${ }^{1,2}$,
Jean-Philippe Collet ${ }^{1}$, Catherine Fargeot ${ }^{3}$,
Gilles Montalescot

> Les révolutions successives ont marqué l'histoire de la cardiologie interventionnelle. La première fut l'introduction de l'angioplastie percutanée par ballonnet, utilisée en alternative à la chirurgie pour la revascularisation coronaire, suivie de l'invention des premiers stents métalliques nus (bare metal stent ou BMS) et leur amélioration en stents dits actifs à élution de médicaments (drug-eluting stent ou DES), réduisant drastiquement le taux de re-sténoses observé avec les BMS. L'amélioration des plates-formes et des polymères des stents (biocompatibles ou résorbables) a permis de réduire le taux de thromboses tardives de stent à des niveaux extrêmement bas. Le développement de stents auto-expansibles est actuellement proposé pour lutter contre le problème de mauvaise apposition tardive aux parois du vaisseau dans l'infarctus du myocarde. Enfin, les nouveaux stents intégralement biorésorbables, pouvant être combinés à des molécules actives antiprolifératives, deviendront très probablement les stents de choix pour la revascularisation coronaire dans un futur proche. <

L'angioplastie percutanée par ballonnet gonflable pour le traitement de la maladie coronaire, développée par Andreas Gruntzig en 1977, a constitué une avancée considérable dans le domaine de la médecine cardiovasculaire. Elle peut être considérée comme la première révolution de la cardiologie interventionnelle. La deuxième révolution a été la création du stent par le Dr Julio Palmaz, qui en a déposé le brevet en 1985, suivie d'une première française, l'implantation du premier stent métallique nu (BMS ou bare metal stent) en 1986 par le Pr Jacques Puel. Le stent a permis de pallier les écueils de la dilatation au ballon et, en particulier, la dissection, le retour élastique (elastic recoil ${ }^{1}$ ) et la réocclusion des vaisseaux traités. L'invention du stent a

${ }^{1}$ Le retour élastique (elastic recoil) désigne le fait qu’après le retrait du ballon la sténose a tendance à se reformer. par exemple).

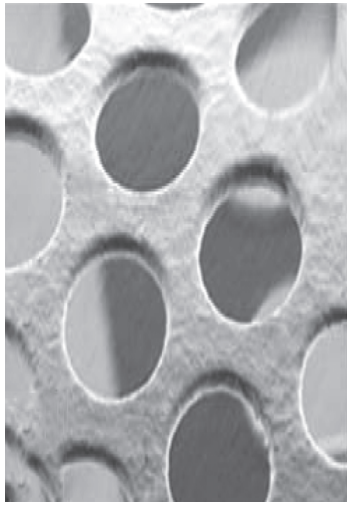

été reconnue par le magazine anglais Intellectual property international magazine comme l'une des dix inventions qui ont changé le monde lors du siècle dernier. La troisième révolution a été l'arrivée, en 2002, du stent dit actif ou à élution de médicaments (DES ou drug-eluting stent), développé avec succès par les laboratoires Johnson \& Johnson, pour réduire le risque de re-sténose observé avec les BMS. Ce risque obligeait a une ré-intervention dans 10 à $20 \%$ des cas et, parfois, conduisait à proposer une revascularisation chirurgicale en cas de re-sténose diffuse ou itérative. Le principe du stent actif à élution de médicament antiprolifératif incorporé au sein d'un polymère le recouvrant a entraîné, de manière parallèle, une augmentation des thromboses tardives de stent, observées avec la première génération de DES, et attribuées à un retard ou à un défaut d'endothélisation du stent. L'amélioration des plates-formes des DES de nouvelle génération a permis de réduire ce taux de thrombose de stent au même niveau que celui du BMS. La diminution de la taille des mailles, et le caractère biocompatible ou résorbable des polymères les recouvrant ont permis de réduire le taux de thromboses tardives de stent à moins de 1 \%. L'utilisation de stents auto-expansibles est actuellement proposée pour éviter la mal-apposition tardive dans l'infarctus du myocarde ${ }^{2}$. La dernière révolution est le stent biorésorbable, qui peut être combiné à des molécules antiprolifératives. Ce concept, encore au stade de validation chez l'homme, va possiblement devenir un standard pour la revascularisation coronaire. Ces stents ont le potentiel théorique de permettre la réduction de la durée de la bithérapie anti-agrégante plaquettaire ${ }^{3}$ et, donc, des complications hémorragiques potentielles

${ }^{2}$ On appelle mal-apposition le fait que le stent n'est pas parfaitement apposé aux parois du vaisseau, laissant un espace.

${ }^{3}$ Association de 2 antiagrégants plaquettaires : acide acétylsalicylique + thiénopyridine (Clopidogrel, 


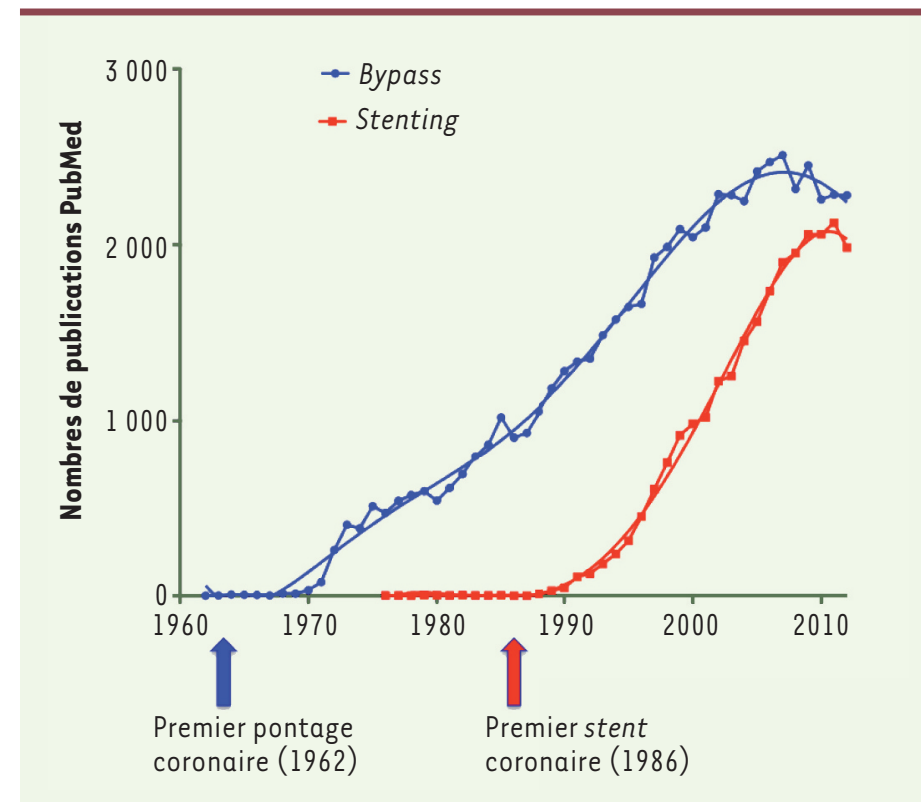

Figure 1. Nombre de publications scientifiques annuelles indexées sur PubMed (http://www.ncbi.nlm.nih.gov/pubmed) pour les recherches « coronary bypass 》 en bleu et « coronary stenting 》 en rouge.

liées à son utilisation, mais aussi la réduction du risque de thrombose tardive de stent. Le développement rapide de cette technique relativement jeune, en comparaison à la chirurgie coronaire, comme l'atteste le nombre de publications indexées sur PubMed (Figure 1), en a fait la technique de première intention dans la plupart des situations cliniques. Elle fait jeu égal avec la chirurgie coronaire pour les patients les plus graves et les lésions les plus sévères.

\section{Les stents actifs ou drug eluting stent (DES) :} architecture du stent de référence actuel

Un stent actif est constitué de trois éléments (Figure 2):

1. une structure métallique (la plate-forme), constituée en général d'un alliage souple d'architecture variable permettant de déployer des forces radiales importantes;

2. un polymère recouvrant la surface du stent, constitué de loges sur la face abluminale (en général) qui permettent de contenir un principe actif :

3. un principe actif, constitué en général d'une molécule antiproliférative, mais aussi d'anticorps ou d'une combinaison de molécules.

\section{La plate-forme}

La plate-forme des stents actuellement utilisés peut avoir l'architecture d'une structure modulaire ou tubulaire. Elle est composée d'acier, d'association de chrome, de cobalt ou d'alliage de cobalt ou encore de platine. D'importantes améliorations des plates-formes utilisées ont été réalisées par les différentes firmes (Figure 3), avec notamment une réduction de l'épaisseur des mailles des stents (ou struts), passant de $140 \mu \mathrm{m}$ à $80 \mu \mathrm{m}$, ce qui permet une meilleure délivrance et améliore ainsi l'accès à des lésions distales et tortueuses. Les études expérimentales ont également démontré une moindre thrombogénicité des mailles fines en comparaison avec les mailles épaisses.

\section{Les polymères}

Les polymères recouvrent la plate-forme du stent et permettent de délivrer, habituellement pendant les deux mois suivant l'implantation, un principe actif dans la paroi artérielle. De très nombreux polymères sont utilisés (phosphorylcholine, copolymère fluoré, acide polylactique, etc.) ; certains d'entre eux, comme l'acide polylactique, sont biodégradables.

\section{Les principes actifs}

La spécificité des DES est d'avoir un principe actif incorporé à leur structure, les distinguant des BMS. La re-sténose intra-stent est le résultat de la prolifération et de la migration de cellules musculaires lisses, et est directement liée à l'implantation du stent. La physiopathologie de la re-sténose est expliquée par l'interaction complexe entre des éléments cellulaires et acellulaires de la paroi artérielle et le sang. Il est désormais démontré que des molécules antiprolifératives et anti-inflammatoires, intégrées à des couches de polymères qui diffusent lentement, induisent une diminution de cette formation néo-intimale. Les deux premières molécules antiprolifératives utilisées chez l'homme ont été le paclitaxel et le sirolimus. Le paclitaxel, un diterpène naturel (provenant de certaines espèces d'if, Taxus brevifolia ou Taxus baccata) ou semi-synthétique, est un poison du fuseau capable de bloquer la division cellulaire en inhibant la dépolarisation des microtubules. II était initialement utilisé comme agent chimiothérapique, et est toujours utilisé dans les cancers du poumon, du sein et de l'ovaire. Le sirolimus (anciennement rapamycine) est un antibiotique de la classe des macrolides initialement développé pour son action immunosuppressive lors des transplantations rénales. II associe une action cytostatique (blocage du cycle cellulaire en phase Gl via le récepteur mTOR [mammalian target of rapamycine]) et anti-inflammatoire. Les résultats des deux études princeps respectives ont été remarquables en termes de taux de re-sténose avec $0 \%$ à six mois dans les deux études (RAVEL [1] pour le CYPHERTM, le premier DES au sirolimus; TAXUS IV [2] pour le TAXUS LIBERTETM, le premier DES au paclitaxel). Pour autant, les résultats de la méta-analyse la plus large dans le domaine [3], portant sur 18023 patients issus de 38 études sur les stents actifs, avec un suivi de quatre ans, ont montré une diminution de $70 \%$ ( $p$ 


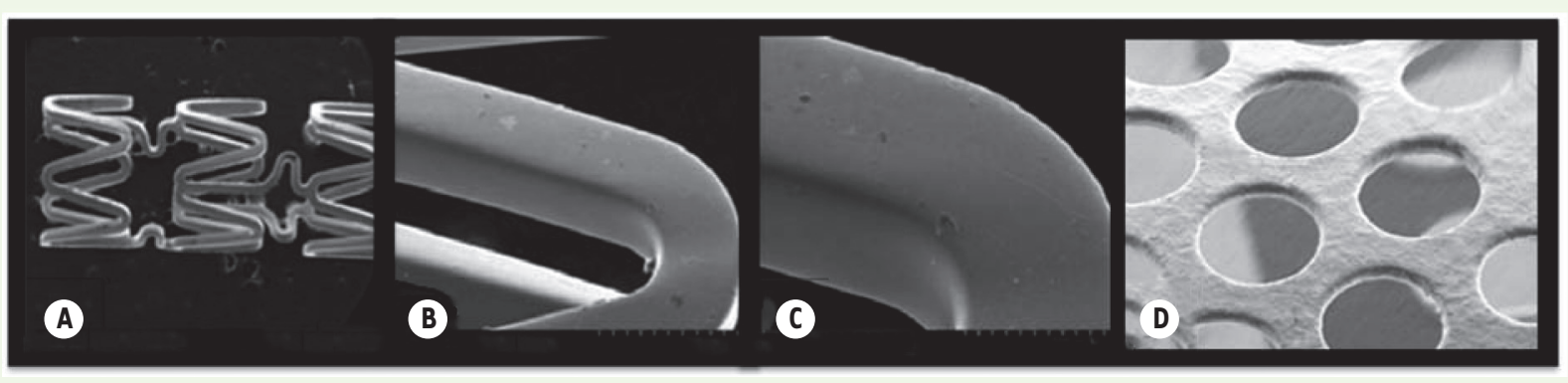

Figure 2. Un stent actif vu au microscope électronique à balayage à différents grossissements.

$<0,0001)$ du taux de revascularisation du vaisseau cible (TLR) pour le stent au sirolimus, et de $58 \%(p<0,0001)$ pour le stent au paclitaxel. Ainsi, par la suite, de nouvelles molécules ont été développées, principalement dérivées du sirolimus qui semble garder l'avantage en termes de réduction de re-sténose. Ces nouvelles molécules sont: le zotarolimus, un analogue semi-synthétique de la rapamycine, dont l'action antiproliférative est très proche de celle du sirolimus, qui bloque aussi le récepteur mTOR et entraîne une inhibition cellulaire en phase Gl ; l'everolimus, un antibiotique de la classe des macrolides, également très proche du sirolimus, lui aussi utilisé comme agent anticancéreux dans le cancer du rein, du sein et les tumeurs neuro-endocrines du pancréas; et le biolimus A9, aussi dérivé du sirolimus, mais dont les caractéristiques de lipophilie importante lui permettent d'être plus rapidement absorbé par la paroi artérielle.

\section{Limites des stents de première génération}

Les premiers stents actifs ont été reçus avec beaucoup d'enthousiasme et, rapidement, leur taux d'utilisation a atteint 50 à $60 \%$ des stents implantés en France. Néanmoins, peu de temps après leur mise sur le marché, des rapports alarmants - issus d'analyses des registres hollandais, suédois et japonais - faisaient état d'une augmentation potentielle de la mortalité, ainsi que du taux de thromboses rapides et tardives constatée avec le stent CYPHER SELECTTM. Ces analyses retrouvaient un taux annuel de thromboses très tardives entre $0,36 \%$ et $0,6 \%$ par an, persistant même au-delà de la cinquième année $[4$, 5]. De plus, des études autopsiques ont montré qu'à 40 mois, $45 \%$ des struts des stents actifs de première génération n'étaient pas correctement ré-endothélialisés, favorisant ainsi les thromboses tardives de stent [6] (Figure 4 A-B).

La deuxième limite des stents expansibles sur ballonnet est leur limite d'adaptation au diamètre de l'artère coronaire, sujette à des phases de vasoconstriction et de vasodilatation selon les situations cliniques. Ainsi, lors d'un infarctus du myocarde, la présence de thrombus et la survenue d'une vasoconstriction sont des phénomènes transitoires qui peuvent exposer à l'existence d'une mal-apposition tardive une fois que le thrombus a été résorbé et que l'artère a récupéré son calibre originel. Ce phénomène de mal-apposition est considéré comme un facteur de thrombose de stent [7] (Figure 4C).

\section{Avantages des stents de nouvelles générations}

Les améliorations des plates-formes des polymères et des principes actifs

Les nouvelles générations de stents actifs (de deuxième, troisième voire quatrième génération) présentent des améliorations sur les trois composants du stent. Les nouvelles plates-formes ont produit des stents plus flexibles dont l'épaisseur des mailles est plus fine. Les modélisations informatiques ont suggéré que l'utilisation de mailles fines s'accompagnerait d'une réduction des turbulences de flux pouvant être à l'origine des phénomènes thrombotiques. Cette moindre thrombogénicité des mailles fines comparée aux mailles épaisses a par la suite été confirmée expérimentalement chez l'animal [8], puis chez l'homme avec un impact majeur de l'épaisseur de la maille du stent sur le risque de thrombose. Néanmoins, l'impact de l'épaisseur de la maille du stent sur le risque de thrombose n'est pas universellement retrouvé, notamment dans les études COMPARE-2 et SORT-OUT VI présentées au congrès Transcatheter cardiovascular therapeutics (TCT) en novembre 2013.

D'importants progrès ont été aussi réalisés au niveau des polymères avec l'utilisation de polymères biocompatibles dont l'épaisseur a été réduite de 6 à $8 \mu \mathrm{m}$ sur certains stents. Certaines endoprothèses, comme les stents BIOMATRIXTM ou NOBORITM, utilisent des polymères biodégradables en 6 à 9 mois, à base d'acide polylactique (PLLA pour acide lactique poly-D, L) ou d'acide polylactique coglycoside (PLGA) pour le stent SYNERGYTM. Enfin, en termes de principes actifs, le sirolimus initialement utilisé a laissé place à ses dérivés (everolimus, zotarolimus) (Tableau I).

De très nombreuses études (Tableau II) ont comparé les stents de nouvelle génération aux stents actifs de première génération. Une récente méta-analyse [9] a comparé les stents actifs à l'everolimus aux stents actifs sans everolimus (essentiellement de première génération) ; elle 


\begin{tabular}{|c|c|c|c|}
\hline & $\begin{array}{l}\text { Principe } \\
\text { actif }\end{array}$ & $\begin{array}{c}\text { Polymère } \\
\text { biodégradable }\end{array}$ & Plate-forme \\
\hline \multicolumn{4}{|l|}{ BMS } \\
\hline TITAN & Non & Non & Acier inox $316 \mathrm{~L}$ \\
\hline DRIVER - INTEGRITY & Non & Non & Alliage cobalt-chrome \\
\hline MULTI-LINK & Non & Non & Alliage cobalt-chrome \\
\hline \multicolumn{4}{|l|}{$1^{\text {re }}$ génération DES } \\
\hline CYPHER & Sirolimus & Non & Acier inox $316 \mathrm{~L}$ \\
\hline TAXUS EXPRESS/LIBERTE & Paclitaxel & Non & Acier inox $316 \mathrm{~L}$ \\
\hline \multicolumn{4}{|l|}{$2^{\mathrm{e}}$ génération DES } \\
\hline ENDEAVOR & Zotarolimus & Non & Alliage cobalt-chrome \\
\hline RESOLUTE & Zotarolimus & Non & Alliage cobalt-chrome \\
\hline XIENCE V/PRIME & Everolimus & Non & Alliage cobalt-chrome \\
\hline \multicolumn{4}{|l|}{$3^{e}$ et $4^{e}$ génération DES } \\
\hline PROMUS ELEMENT & Everolimus & Non & Alliage platine-chrome \\
\hline SYNERGY & Everolimus & Oui & Alliage platine-chrome \\
\hline NOBORI & Biolimus A9 & Oui & Acier inox $316 \mathrm{~L}$ \\
\hline BIOMATRIX FLEX & Biolimus A9 & Oui & Acier inox $316 \mathrm{~L}$ \\
\hline COMBO & $\begin{array}{c}\text { Sirolimus + } \\
\text { anticorps } \\
\text { anti-CD34 }\end{array}$ & Oui & Acier inox $316 \mathrm{~L}$ \\
\hline \multicolumn{4}{|l|}{$1^{\text {re }}$ génération BDS } \\
\hline IGAKI-TAMAI & Non & Oui & Biorésorbable (PLLA) \\
\hline REVA & Non & $\begin{array}{l}\text { Oui } \\
\text { (radio-opaque) }\end{array}$ & Biorésorbable (AA L-tyrosine) \\
\hline ART & Non & Oui & Biorésorbable (PLLA) \\
\hline \multicolumn{4}{|l|}{$2^{\mathrm{e}}$ génération BDES } \\
\hline BVS & Everolimus & Oui & Biorésorbable (PLLA) \\
\hline AMS-3 & Sirolimus & Oui & Biorésorbable (magnésium) \\
\hline DREAMS & Paclitaxel & Oui & Biorésorbable (magnésium) \\
\hline IDEAL (2.0) & Sirolimus & Oui & $\begin{array}{l}\text { Biorésorbable } \\
\text { (poly[anhydride-ester]) }\end{array}$ \\
\hline RESOLVE & Sirolimus & $\begin{array}{c}\text { Oui } \\
\text { (radio-opaque) }\end{array}$ & Biorésorbable (PLLA) \\
\hline BIOMINE & Sirolimus & Oui & Biorésorbable (PLLA + PLGA) \\
\hline
\end{tabular}

Tableau I. Principaux constituants des différents stents. BMS : bare metal stent; DES : drug-eluting stent ; BDS : biodegradable stents; BDES : biodegradable eluting stents. a porté sur 17101 patients et 13 essais randomisés. Cette étude a confirmé, d'une part, une diminution du taux de revascularisation du vaisseau cible de $33 \%(p=0,001)$ et, d'autre part, une meilleure sécurité avec une réduction de $45 \%$ du risque relatif de thrombose de stent $(p=0,004)$. Une autre métaanalyse, publiée en 2012, a comparé les stents actifs aux stents nus, mais aussi les stents actifs entre eux [10]; elle a porté sur 76 essais et 117762 patients. Elle a retrouvé une nette supériorité des stents actifs par rapport aux stents nus, pour réduire le risque de revascularisation du vaisseau cible. Elle a montré un avantage en termes d'efficacité en faveur des stents au sirolimus, à l'everolimus et au zotarolimus, alors qu'en termes de sécurité (risque de thrombose de stent), les stents à l'everolimus semblaient les plus sûrs. Les stents au biolimus (BIOMATRIXTM) ont été comparés aux stents au sirolimus (CYPHER SELECTTM) dans une étude de non-infériorité portant sur 1707 patients [11]. Les résultats à quatre ans de cette étude ont montré une non-infériorité atteinte, et même une supériorité, sur les critères décès cardiovasculaire, infarctus et revascularisation du vaisseau cible par rapport aux stents au sirolimus; $18,7 \%$ biolimus versus $22,6 \%$ sirolimus, $p$ pour non-infériorité < 0,0001, p pour supériorité $=0,05$ [12]. II a été observé une réduction de $38 \%$ du risque relatif de thrombose tardive de stent $(p=0,09)$ avec une réduction des thromboses très tardives (> un an) entre la première et la quatrième année $(p=0,004)$. Les résultats des différentes études avec les stents au polymère biodégradable sont en faveur d'une réduction des thromboses de stent en comparaison avec les stents au sirolimus.

\section{Les stents entièrement résorbables}

Les stents entièrement biorésorbables offrent plusieurs avantages potentiels sur les BMS ou DES, y compris 


\section{Épaisseur de maille}

Épaisseur du polymère

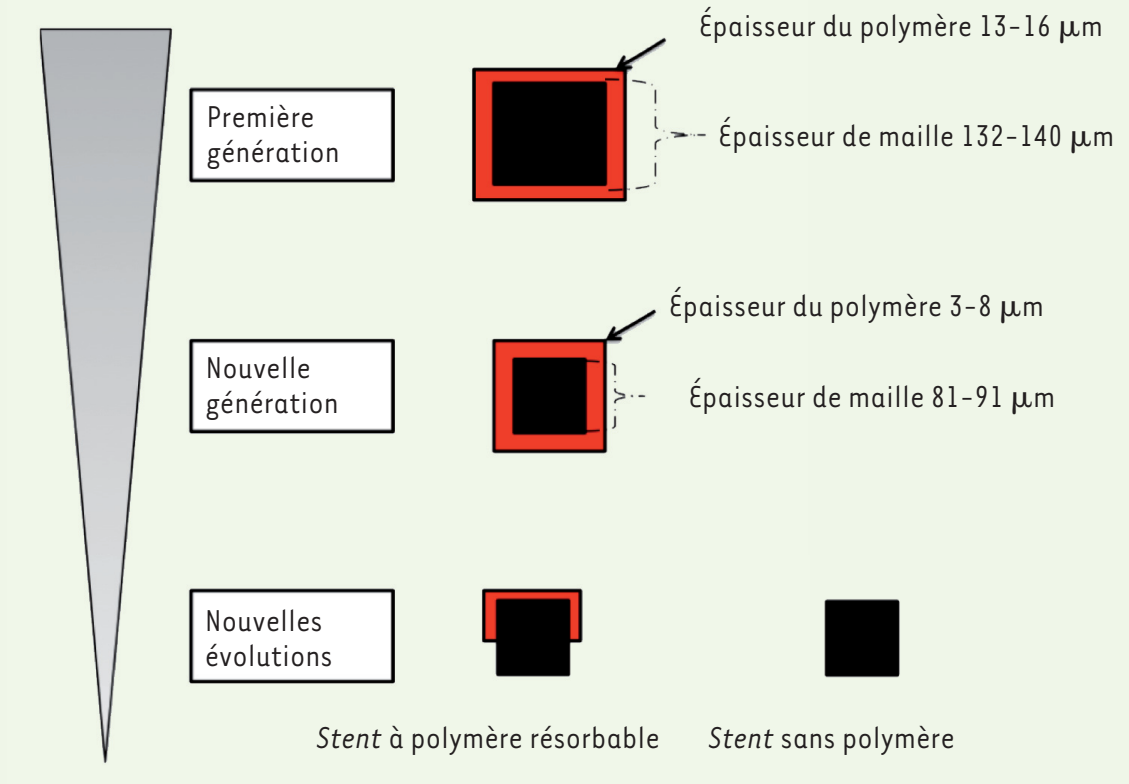

Figure 3. Améliorations techniques des stents actifs entre la première et la dernière génération.

[13]. Le stent Bioresorbable vascular scaffold (BVSTM ou ABSORBTM) est recouvert d'une couche d'everolimus intégrée à une matrice de PDLLA dont la diffusion se fait dans les premiers mois, la résorption complète du polymère se faisant en 24 mois. Les premières études cliniques avec le BVS de première génération étaient encourageantes, avec cependant une perte du diamètre intraluminal (late loss) plutôt élevée [14]. Le BVS de deuxième génération (Revision 1.1) a permis d'obtenir une meilleure force radiale et un meilleur late loss [15]. Le stent métallique AMS-1 (absorbable metallic stent) est composé de $93 \%$ de magnésium et de $7 \%$ de les nouveaux DES avec polymère dégradable. La disparition du stent limiterait la durée de libération de substances actives pour éliminer les défauts d'endothélialisation des mailles ou la persistance du polymère, qui sont autant de facteurs de risque de thrombose de stent, limitant ainsi la durée d'une double thérapie anti-agrégante plaquettaire et les complications hémorragiques qui lui sont associées. La disparition du matériel métallique est supposée restituer les propriétés vasomotrices de la paroi artérielle et la capacité du vaisseau à s'adapter aux contraintes de cisaillement. Enfin, la résorption complète de la prothèse permettrait, une fois le stent résorbé, de récupérer un accès aux branches collatérales et d'envisager tout type de revascularisation future (chirurgicale ou instrumentale) sans limitation. Néanmoins, La conception de telles endoprothèses se heurte à trois obstacles potentiels: l'absence de radio-opacité qui nécessite le recours à des marqueurs radio-opaques, une force radiale réduite comparée aux stents métalliques entraînant la conception de mailles plus épaisses, et enfin une capacité de déformation limitée.

Les stents biorésorbables utilisent, soit des polymères comme l'acide polylactique (PLLA) ou l'anhydride d'acide salicylique et d'acide adipique, soit des alliages métalliques à base de magnésium (stent DREAMSTM). Le polymère le plus largement utilisé actuellement est le PLLA. Le LLA est un acide qui est produit naturellement dans le corps humain et le PLLA est métabolisé en approximativement 12 à 18 mois avec libération de dioxyde de carbone et d'eau. Le premier stent biorésorbable en PLLA implanté chez l'homme a été le stent japonais IGAKI$T_{\text {TAMAITM }}{ }^{T M}$ dont le suivi clinique à près de 10 ans dans une petite série de patients $(n=50)$ a été récemment publié. L'étude montre des résultats cliniques satisfaisants, comparables au stent nu, mais dont le principal écueil était la longueur du temps de résorption, proche de trois ans métaux rares. Les travaux expérimentaux ont montré une dégradation complète du magnésium en sels non organiques sur une période de deux mois [16]. Deux évolutions techniques de ce stent ont été réalisées afin, d'une part, d'améliorer la force radiale et, d'autre part, d'y incorporer une matrice biorésorbable permettant la libération de substance active dans la paroi (AMS-2 puis AMS-3). Les études cliniques évaluant son efficacité et sa sécurité d'emploi sont en cours. La société française ART développe un stent biorésorbable en polymère d'acide lactique dont la dégradation débute vers trois mois $[17,18]$. Après le premier patient implanté en 2012, une étude clinique sur des lésions de novo est prévue en 2013.

Le stent IDEAL ${ }^{T M}$ est unique dans le sens où le polymère qui le compose est un poly(anhydride-ester) composé d'un trimère de deux molécules d'acide salicylique reliées par un acide gras, et d'un enrobage en sirolimus permettant d'obtenir des effets antiprolifératifs et anti-inflammatoires. Lors de l'implantation, ce stent a une résistance radiale significativement plus élevée que celle du stent CYPHERTM en raison de sa structure presque cylindrique (la surface de l'artère occupée par le stent est de $65 \%$ ), mais avec une dégradation en 50 jours environ; la résistance radiale est équivalente à celle de CYPHERTM. La dégradation complète est observée en 12-15 mois. La première étude montrant de bons résultats a démontré que l'hyperplasie néo-intimale n'a pas été suffisamment supprimée [19]. Une seconde 


\begin{tabular}{|c|c|c|c|c|}
\hline $\begin{array}{l}\text { Principe } \\
\text { actif }\end{array}$ & Études & $\begin{array}{l}\text { Nombre de } \\
\text { patients }\end{array}$ & Comparaison & $\begin{array}{c}\text { Type } \\
\text { d'étude }\end{array}$ \\
\hline \multirow{6}{*}{ Everolimus } & SPIRIT II & 300 & XIENCE V : TAXUS & Supériorité \\
\hline & SPIRIT III & 1002 & XIENCE V : TAXUS EXPRESS & Supériorité \\
\hline & SPIRIT IV & 3690 & XIENCE V : TAXUS EXPRESS & Supériorité \\
\hline & COMPARE & 1800 & XIENCE V : TAXUS LIBERTE & Supériorité \\
\hline & PLATINIUM & 1530 & $\begin{array}{l}\text { PROMUS/XIENCE V : PRO- } \\
\text { MUS ELEMENT }\end{array}$ & Non-infériorité \\
\hline & SORTOUT IV & 2774 & $\begin{array}{l}\text { XIENCE V/PROMUS : CYPER } \\
\text { SELECT }\end{array}$ & Non-infériorité \\
\hline \multirow{7}{*}{ Zotarolimus } & $\begin{array}{l}\text { ENDEAVOR } \\
\text { III }\end{array}$ & 436 & ENDEAVOR : CYPHER & Supériorité \\
\hline & $\begin{array}{l}\text { ENDEAVOR } \\
\text { IV }\end{array}$ & 1548 & ENDEAVOR : TAXUS & Supériorité \\
\hline & ZEST & 2645 & ENDEAVOR : TAXUS/CYPHER & $\begin{array}{l}\text { Non-infériorité/ } \\
\text { supériorité }\end{array}$ \\
\hline & PROTECT & 8800 & ENDEAVOR : CYPHER & Supériorité \\
\hline & SORTOUT III & 2323 & ENDEAVOR : CYPHER & Supériorité \\
\hline & RESOLUTE & 2292 & RESOLUTE : XIENCE V & Non-infériorité \\
\hline & TWENTE & 1391 & RESOLUTE : XIENCE V & Non-infériorité \\
\hline \multirow{3}{*}{ Biolimus } & LEADERS & 1707 & BIOMATRIX : CYPHER SELECT & Non-infériorité \\
\hline & NOBORI 1 & 243 & NOBORI : TAXUS LIBERTE & Supériorité \\
\hline & COMPAREII & 2707 & NOBORI : XIENCE V PROMUS & Non-inferiorité \\
\hline
\end{tabular}

Tableau II. Principales études publiées comparant les stents actifs de nouvelle génération avec les stents actifs de première génération.

version de ce stent a été développée depuis, dont la géométrie est moins cylindrique et l'épaisseur de maille moindre, mais la dose de sirolimus augmentée et la cinétique de relargage diminuée. Le stent REVATM est un stent en polycarbonate qui se dégrade en eau, dioxyde de carbone et éthanol, avec une durée de résorption d'approximativement trois ans. II s'agit d'une endoprothèse radio-opaque, pourvue d'une bonne flexibilité et d'une force radiale satisfaisante. Les premières études cliniques ont montré des résultats superposables à celui d'un stent conventionnel, mais avec un taux de revascularisation très élevé lié à la fragilisation et à la fragmentation du polymère. Un stent de deuxième génération, le REZOLVETM, pourvu d'un polymère plus robuste et d'un coating au sirolimus, est en cours d'évaluation (Figure 4).

\section{Un stent auto-expansible pour l'infarctus}

Les tout premiers stents (WALL STENT) étaient des stents autoexpansibles. Après avoir été surclassés par les stents à expansion par ballonnet, ils sont de nouveau proposés dans l'infarctus du myocarde (STENTYSTM) pour diminuer le risque de mal-apposition constaté avec les stents actifs non biorésorbables [20] (Figure 5).
La mal-apposition est un facteur de risque de thrombose tardive intra-stent. L'utilisation d'une plate-forme en nitinol, un alliage de nickel et de titane ayant comme spécificité d'avoir une mémoire de forme et une super-élasticité, permet d'épouser parfaitement la paroi de l'artère et de s'adapter à son diamètre, une fois les phénomènes de vasoconstriction terminés et/ou après la disparition des thrombus parfois apposés entre la paroi et le stent. Cette situation est particulièrement fréquente en cas d'infarctus du myocarde. Les stents nus en nitinol de première génération ont montré de bons résultats dans les premières études cliniques (APPOSITION IV). Ils sont évalués dans leur nouvelle version enrobée de sirolimus en comparaison avec les stents actifs de deuxième génération (APPOSITION V).

\section{Conclusion}

Les stents ont révolutionné la cardiologie interventionnelle et sont devenus des outils thérapeutiques de premier choix dans le traitement de la revascularisation coronaire. Ils ont résolu les écueils de l'angioplastie au ballonnet (dissection, ré-occlusion précoce), et les stents à élution de médicaments ont permis de vaincre la re-sténose et la thrombose très tardive, notamment avec l'amélioration des polymères et des plates-formes pour les stents de nouvelles générations, en parallèle des progrès réalisés en pharmacologie antithrombotique. Les polymères ou stents biorésorbables ont clairement pour objectif de faire disparaitre les risques résiduels, à savoir la mal-apposition, la thrombose de stent tardive, tout en permettant de réduire la durée de traitements anti-agrégants. Définir avec précision la place des nouveaux stents biorésorbables dans l'arsenal thérapeutique de l'angioplastie coronaire est un enjeu majeur. L'évaluation de leurs performances dans le traitement des lésions tortueuses, complexes et calcifiées, ou dans le cadre des syndromes coronaires aigus reste à faire. $\varepsilon$ n attendant, les stents actifs non résorbables de dernières générations sont la référence pour l'angioplastie coronaire en l'absence de contre-indications. $\diamond$ 


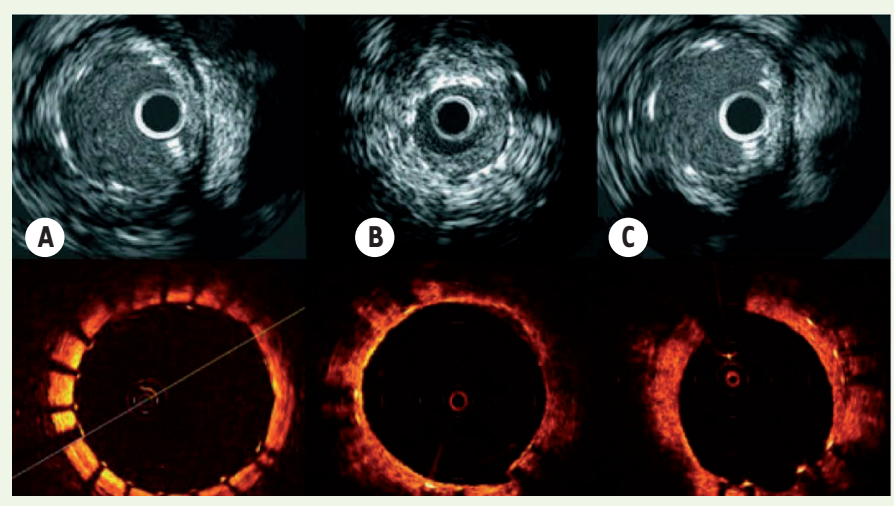

Figure 4. Analyse par imagerie d'un stent implanté de type IVUS (haut) ou OCT (bas). A. Implantation d'un stent avec expansion optimale (IVUS en haut et OCT en bas), mais absence de recouvrement des struts par l'endothélium (phase initiale). $\boldsymbol{B}$. Formation néo-intimale recouvrant les struts du stent avec légère réduction du calibre de la lumière. $C$. Mal-apposition avec struts non recouverts et débordant dans la lumière de l'artère.

\section{SUMMARY}

\section{Coronary stents: 30 years of medical progress}

The history of interventional cardiology has been marked by several technologic revolutions since the late 1970s. The first key step was the use of inflatable balloon angioplasty as an alternative to CABG surgery for coronary revascularization, followed by intracoronary delivery of bare metal stent (BMS) and drug eluting stents (DES) to drastically reduce intracoronary restenosis observed with BMS. Improved stents platforms and polymers (absorbable or biocompatible) led to a dramatic reduction in the rate of late stent thrombosis. Self-expanding stents are now available to improve stent aposition especially in acute myocardial infarction. The emergence of new fully bioabsorbable stents that can be combined with antiproliferative drugs is the ongoing revolution. A new generation of stents is continuously improving and likely to become the ideal stent for coronary revascularization in the near future. $\diamond$

\section{LIENS D'INTÉRÊT}

J. Silvain has received research grants from Sanofi-Aventis, Daiichi-Sankyo, Eli Lilly, Inserm, Fédération française de cardiologie and Société française de cardiologie ; consultant fees from Correvio, Daiichi-Sankyo and Eli Lilly; and lecture fees from AstraZeneca, $A A Z$, Boehringer-Ingelheim, Cordis, Daiichi-Sankyo, Eli Lilly, Stentys and the Medicines company. G. Cayla reports receiving consulting fees from AstraZeneca, Eli Lilly, DaiichiSankyo, and Abbott, and lecture fees from Astra Zeneca, Eli Lilly, Daiichi-Sankyo, Abbott Vascular, Bristol-Myers Squibb, Bayer, Boehringer Ingelheim, CSL Behring, Iroko Cardio International, Pfizer, and Novartis. J.-P. Collet has received research grants from BristolMyers Squibb, Sanofi-Aventis, Eli Lilly, Guerbet Medical, Medtronic, Boston Scientific, Cordis, Stago, Centocor, Fédération française de cardiologie, and Société française de cardiologie; consulting fees from Sanofi-Aventis, Eli Lilly, and Bristol-Myers Squibb; and lecture fees from Bristol-Myers Squibb, Sanofi-Aventis, and Eli Lilly. G. Montalescot reports receiving research grants to the institution or consulting/lecture fees from Abbott Vascular, Asante, AstraZeneca, Atrium, Bayer, Biotronik, BMS, Boehringer-Ingelheim, Boston Scientific, Choice Pharma, Brahms, CCS, CHUV, Cordis, Daiichi-Sankyo, Duke Institute, Eli-Lilly, Europa, EuroRSCG, Fédération française de cardiologie, Fondation de France, GLG, GSK, HUG, Indegene, Institut de France, Iroko, Lead-up, Medtronic, McKinsey,

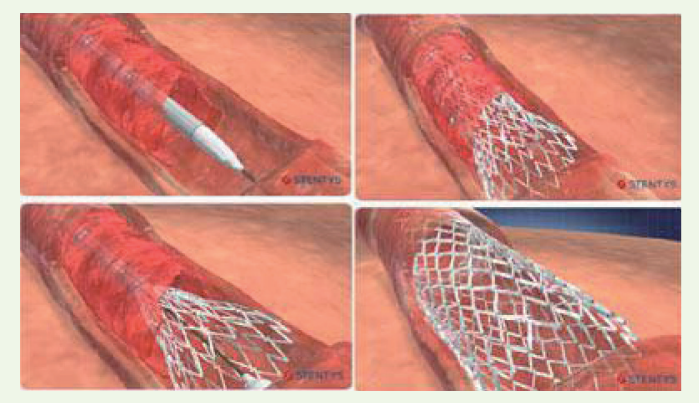

Figure 5. Stent STENTYS auto-expansible.

MSD, Nanospheres, Navigant, Novartis, Pfizer, Portola, Roche, Royal College Physicians, Sanofi-Aventis, Stentys, SGAM, Société française de cardiologie, Springer, Thrombosis research institute, the Medicines company, TIMI group, US Zurich, WebMD, Wolters. C. Fargeot: pas de lien d'intérêt.

\section{RÉFÉRENCES}

1. Morice MC, Serruys PW, Sousa JE, et al. A randomized comparison of a sirolimus-eluting stent with a standard stent for coronary revascularization. N Engl J Med 2002 ; 346 : 1773-80.

2. Stone GW, Ellis SG, Cox DA, et al. A polymer-based, paclitaxel-eluting stent in patients with coronary artery disease. $N$ Engl J Med 2004 ; $350: 221-31$.

3. Stettler C, Wandel S, Allemann S, et al. Outcomes associated with drugeluting and bare-metal stents: a collaborative network meta-analysis. Lancet 2007 ; 370 : 937-48.

4. Raber L, Magro M, Stefanini GG, et al. Very late coronary stent thrombosis of a newer-generation everolimus-eluting stent compared with earlygeneration drug-eluting stents: a prospective cohort study. Circulation $2012 ; 125: 1110-21$.

5. Kimura T, Morimoto T, Nakagawa Y, et al. Very late stent thrombosis and late target lesion revascularization after sirolimus-eluting stent implantation: five-year outcome of the j-Cypher Registry. Circulation 2012 ; 125 : 584-91.

6. Cook S, Wenaweser P, Togni M, et al. Incomplete stent apposition and very late stent thrombosis after drug-eluting stent implantation. Circulation $2007 ; 115: 2426-34$.

7. Hassan AK, Bergheanu SC, Stijnen T, et al. Late stent malapposition risk is higher after drug-eluting stent compared with bare-metal stent implantation and associates with late stent thrombosis. Eur Heart J 2010 ; $31: 1172-80$.

8. Kolandaivelu K, Swaminathan R, Gibson WJ, et al. Stent thrombogenicity early in high-risk interventional settings is driven by stent design and deployment and protected by polymer-drug coatings. Circulation 2011 ; $123: 1400-9$.

9. Baber U, Mehran R, Sharma SK, et al. Impact of the everolimus-eluting stent on stent thrombosis: a meta-analysis of 13 randomized trials. J Am Coll Cardiol $2011 ; 58: 1569-77$.

10. Bangalore $S$, Kumar S, Fusaro M, et al. Short- and long-term outcomes with drug-eluting and bare-metal coronary stents: a mixed-treatment comparison analysis of 117762 patient-years of follow-up from randomized trials. Circulation 2012; $125: 2873-91$.

11. Windecker S, Serruys PW, Wandel S, et al. Biolimus-eluting stent with biodegradable polymer versus sirolimus-eluting stent with durable polymer for coronary revascularisation (LEADERS): a randomised non-inferiority trial. Lancet 2008 ; 372 : 1163-73.

12. Stefanini GG, Kalesan B, Serruys PW, et al. Long-term clinical outcomes of biodegradable polymer biolimus-eluting stents versus durable polymer sirolimus-eluting stents in patients with coronary artery disease (LEADERS): 4 year follow-up of a randomised non-inferiority trial. Lancet $2011 ; 378$ : 1940-8.

13. Nishio S, Kosuga K, Igaki K, et al. Long-term (> 10 years) clinical outcomes of first-in-human biodegradable poly-I-lactic acid coronary stents: IgakiTamai stents. Circulation 2012 ; 125 : 2343-53. 


\section{RÉFÉRENCES}

14. Ormiston JA, Serruys PW, Regar $\varepsilon$, et al. A bioabsorbable everolimus-eluting coronary stent system for patients with single de-novo coronary artery lesions (ABSORB): a prospective open-label trial. Lancet 2008 ; $371: 899-907$.

15. Okamura T, Garg S, Gutierrez-Chico JL, et al. In vivo evaluation of stent strut distribution patterns in the bioabsorbable everolimus-eluting device: an OCT ad hoc analysis of the revision 1.0 and revision 1.1 stent design in the ABSORB clinical trial. Eurolntervention $2010 ; 5: 932-8$.

16. Waksman R. Current state of the absorbable metallic (magnesium) stent. Eurolntervention 2009; 5 (suppl F) : F94-7.

17. Lafont A, Durand $\varepsilon$. ART: concept of a bioresorbable stent without drug elution. Eurolntervention $2009 ; 5$ (suppl F) : F83-7.

18. Durand $\varepsilon$, Lemitre $M$, Couty L, et al. Adjusting a polymer formulation for an optimal bioresorbable stent: a 6 -month follow-up study. Eurolntervention 2012 ; 8 : 242-9.
19. Jabara R, Pendyala L, Geva S, et al. Novel fully bioabsorbable salicylatebased sirolimus-eluting stent. Eurolntervention 2009 ; 5 (suppl F) : F58-64.

20. Van Geuns RJ, Tamburino C, Fajadet J, et al. Self-expanding versus balloon-expandable stents in acute myocardial infarction: results from the APPOSITION II study: self-expanding stents in ST-segment elevation myocardial infarction. JACC Cardiovasc Interv $2012 ; 5$ : 1209-19.

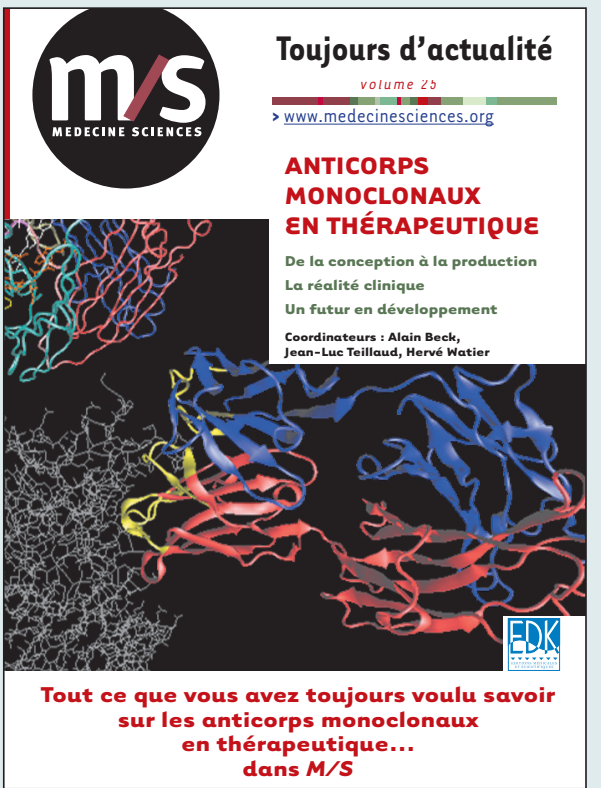

Tout ce que vous avez toujours voulu savoir sur les anticorps monoclonaux en thérapeutique... dans Médecine/Sciences. Pourquoi un numéro spécial de Médecine/Sciences sur les anticorps monoclonaux thérapeutiques? II nous a semblé que le moment était venu de dresser un état des lieux de ces biomédicaments qui prennent désormais une place considérable - et croissante dans les traitements de maladies souvent lourdes et désespérantes. Ce voyage que nous vous proposons à la découverte du monde des anticorps thérapeutiques nous a appris, ou plutôt rappelé, une évidence : les compétences en France sont fortes et nombreuses, qu'elles soient académiques ou industrielles, biotechnologiques ou cliniques. Le paysage français, trop longtemps discret, bruisse désormais de mille initiatives balayant de multiples aspects des anticorps thérapeutiques : études précliniques et cliniques menées avec de nouveaux anticorps dirigés contre des cibles originales, développement de nouveaux formats d'anticorps ou d'anticorps optimisés reposant sur des études structurales et fonctionnelles sophistiquées, recherche active de cibles pertinentes, mise au point de méthodologies de bioproduction, de couplage, etc. L'expansion industrielle rapide de ce champ est un défi que peut et doit relever notre pays, défi tant scientifique qu'économique, avec ses combats pour la propriété intellectuelle et pour l'emploi de nos jeunes scientifiques.

Alain Beck, Jean-Luc Teillaud, Hervé Watier

À retourner à EDK, 25, rue Daviel - 75013 Paris, France

Tél. : 0158101905 - Fax : 0143293262 - E-mail : edk@edk.fr

NOM :

Prénom :

Adresse :

Code postal :

Ville :

Pays :

Fonction :

Je souhaite recevoir $\mathbf{M} / \mathbf{S} \mathbf{n}^{\circ} \mathbf{1 2}$ - décembre 2009 (Anticorps monoclonaux en thérapeutique) : $25 €+3 €$ de port $=\mathbf{2 8} €$ TTC en . exemplaire, soit un total de $€$

Par chèque, à l'ordre de $\mathbf{E} \mathbf{D} \mathbf{K}$

Par carte bancaire : $\square$ Visa

Eurocard/Mastercard

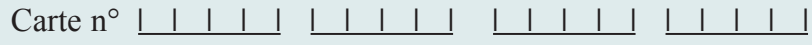

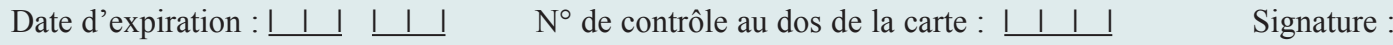

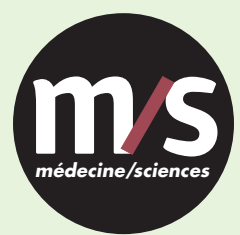

Tarifs d'abonnement $m / s-2014$

Abonnez-vous

à médecine/sciences
$>$ Grâce à $m / s$, vivez en direct les progrès des sciences biologiques et médicales

\section{Bulletin d'abonnement} page 334 dans ce numéro de $\mathrm{m} / \mathrm{s}$

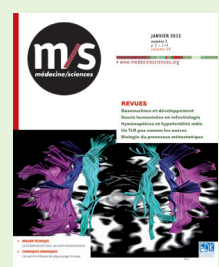

